\title{
Near-Field Mid-Infrared Plasmonics in Complex Nanostructures with Monochromated Electron Energy Loss Spectroscopy
}

Jordan A. Hachtel ${ }^{1}$, Juan Carlos Idrobo ${ }^{1}$, Roderick B. Davidson $\mathrm{II}^{2}$, Richard F. Haglund ${ }^{3}$, Sokrates T. Pantelides $^{3,4,5}$ and Benjamin J. Lawrie ${ }^{6}$

${ }^{1 .}$ Center for Nanophase Materials Sciences, Oak Ridge National Laboratory, Oak Ridge, TN USA

2. Chemical Division, Naval Research Laboratory, Annapolis, MD USA

${ }^{3 .}$ Materials Science and Technology Division, Oak Ridge National Laboratory, Oak Ridge, TN USA

4. Department of Physics and Astronomy, Vanderbilt University, Nashville, TN USA

${ }^{5 .}$ Department of Electrical Engineering \& Computer Science, Vanderbilt University Nashville, TN USA

${ }^{6 .}$ Computational Sciences \& Engineering Division, Oak Ridge National Laboratory, Oak Ridge, TN USA

Localized surface plasmon resonances (LSPRs) in complex nanostructures have seen widespread use in bringing optical phenomena to on-chip applications [1]. For dipolar excitations in symmetric nanoparticles of electron dense materials like $\mathrm{Au}$, Ag, or Al, LSPRs are usually in the visible range. However, in complex nanostructures composed of those materials, unique IR and telecom plasmon resonances can emerge [2].

The nanoscale confinement of light to the surface of a structure makes LSPRs highly versatile in nanotechnology, however the confinement also makes them difficult to study at the near-field through traditional optical methods due to the diffraction limit. Scanning transmission electron microscopy (STEM) and electron energy loss spectroscopy (EELS) can be used to map plasmon resonances directly at the nanoscale, but the inherent energy spread of the electron probe has long made plasmon modes in the IR spectrum difficult to observe. However, recent advances in monochromation have greatly increased the energy resolution of STEM-EELS and greatly reduced the background in the IR [3].

Here we examine Au Archimedean nanospirals. The structures have strong potential for on-chip applications due to the highly-tunable non-centrosymmetric geometry that supports many unique plasmon modes, as well as producing many interesting optical phenomena such as second harmonic generation and super-continuum luminescence [4,5]. The plasmon modes extend across a broad spectral range and many are primarily active in the IR, making the structure ideal for monochromated EELS.

Figure 1a shows a high angle annular dark field (HAADF) image of a Au nanospiral with a winding number of $5 \pi$, arm width and spacing of $80 \mathrm{~nm}$, and a Au film thickness of $40 \mathrm{~nm}$. A spectrum image (SI) of the nanospiral is acquired with a Nion monochromated, aberration-corrected UltraSTEM operated at $60 \mathrm{kV}$ [6]. Monochromation is performed by using a variable slit to balance the energy resolution with the probe current. To maximize signal-to-noise while minimizing the energy spread in the probe, the slit is adjusted so that the zero loss peak has a full-width half-maximum of $75 \mathrm{meV}$.

Two distinct peaks are observed in the IR range from the SI, at $440 \mathrm{meV}$ and at $260 \mathrm{meV}$, which corresponds to wavelengths of $2815 \mathrm{~nm}$ and $4765 \mathrm{~nm}$ respectively, and each exhibiting a completely different spatial profile. The ability to map complex mid-IR plasmon modes with a monochromated aberration corrected STEM opens new avenues to explore on-chip optical technologies with subÅngstrom spatial resolution [7]. 
References:

[1] A.E. Cetin et al, Light: Science and Applications 3 (2014) e122

[2] X. Guo et al, Physical Review Letters 117 (2016) p. 123902

[3] O. L. Krivanek et al, Nature 514 (2014) p. 209

[4] J. I. Ziegler and R.F. Haglund, Nano Letters 10 (2010) p. 3013

[5] A.V. Krassavin et al, Nature Communications 7 (2016) 11497

[6] O. L. Krivanek et al, Phil. Trans. R. Soc. A 367 (2009) p. 3683

[7] Research supported by ORNL's Center for Nanophase Materials Sciences, which is a U.S. DOE

Office of Science User Facility; the U.S. DOE Office of Science, Basic Energy Sciences, Materials

Science and Engineering Division; by ORNL's Laboratory Directed Research and Development

Program, managed by UT-Battelle, LLC, for the U.S. DOE; U.S. DOE grants DE-FG02-09ER46554, DE-FG02-01ER45916, the McMinn Endowment at Vanderbilt University.
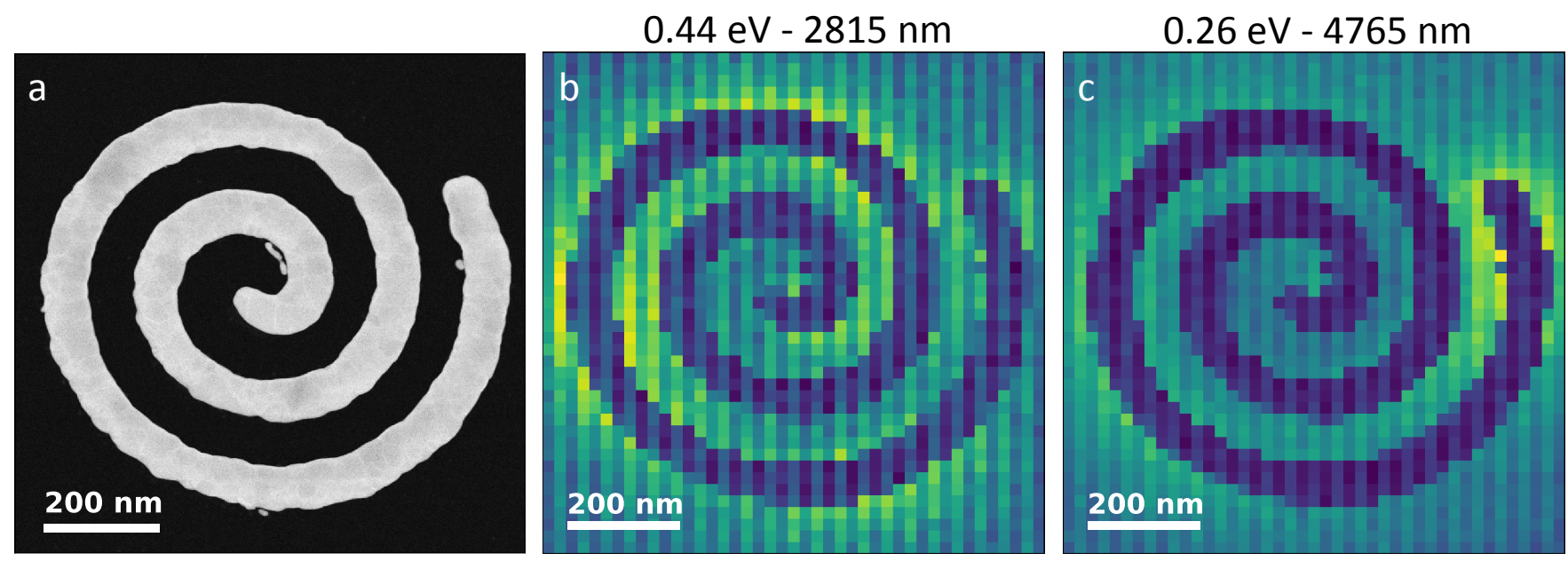

Figure 1. (a) HAADF-STEM image of the Archimedean Au nanospiral. (b) and (c) Spectrum images of distinct plasmon modes in the Mid-IR, at $2815 \mathrm{~nm}$ (b) and $4765 \mathrm{~nm}$ (c) 